\title{
THE CONTRIBUTION OF ATMOSPHERIC AEROSOLS TO THE MARTIAN OPPOSITION EFFECT*
}

\author{
JAYLEE M. MEAD \\ Laboratory for Space Physics, NASA Goddard Space Flight Center, Greenbelt, Md., U.S.A.
}

\begin{abstract}
Mie scattering calculations have been made for atmospheric aerosols having various indices of refraction to determine their possible contribution to a Martian opposition effect, such as that reported by O'Leary in 1967. Neither substances with a real index between 1.20 and 1.50, such as ice, water, or solid $\mathrm{CO}_{2}$, nor highly absorbing materials, such as limonite, can produce the observed effect. Submicron-sized spherical particles with refractive indices of 1.55 to 2.00 do, on the other hand, exhibit a marked increase in reflectivity at small phase angles and might be responsible for the enhanced brightness at the shorter wavelengths.
\end{abstract}

Observations of Mars made by O'Leary (1967a, b) during the 1967 opposition show an 'opposition effect', i.e., a non-linear surge in brightness as the planet approaches $0^{\circ}$ phase angle (the angle at the planet between the directions to the sun and to the observer). Figure 1, based on data taken from O'Leary and Rea (1968), shows the observed reflectivities, adjusted for the color of the sun, as a function of phase angle $\alpha$, for five colors: $U, B, V, R$, and $I$. The observations were made at phase angles of $1.2^{\circ}$ to $8^{\circ}$ and are indicated by solid lines. These were fitted to the linear phase functions for $\alpha \geqslant 10^{\circ}$, reported by de Vaucouleurs (1964).

As O'Leary and Rea pointed out, the opposition effect is much more pronounced at shorter wavelengths than at longer wavelengths, as evidenced by the fact that the $U$ and $B$ observations depart much more from the linear extrapolation than do the curves at $R$ and $I$. The reflectivity, or albedo, on the other hand, is much greater at longer wavelengths than at shorter ones.

This increased opposition effect in the blue and ultraviolet could be primarily a surface effect in that the surface may have a much greater increase in reflectivity at shorter wavelengths; alternatively, it could be primarily due to light scattering in the atmosphere, as suggested by O'Leary (1967a).

Rayleigh scattering by molecules and by particles small compared to the wavelength of observation does not provide a sudden increase of brightness near $0^{\circ}$ phase angle. Therefore, if the effect is primarily atmospheric, particles of larger size must be responsible.

Because of the low albedo in the $U$, a small brightness contribution by atmospheric aerosols will have a comparatively large effect; in the $I$, where the surface is much brighter, a small brightness contribution by aerosols will cause little or no change in the total brightness.

* The complete version of this paper appears in Icarus 13, No. 1 (1970). 


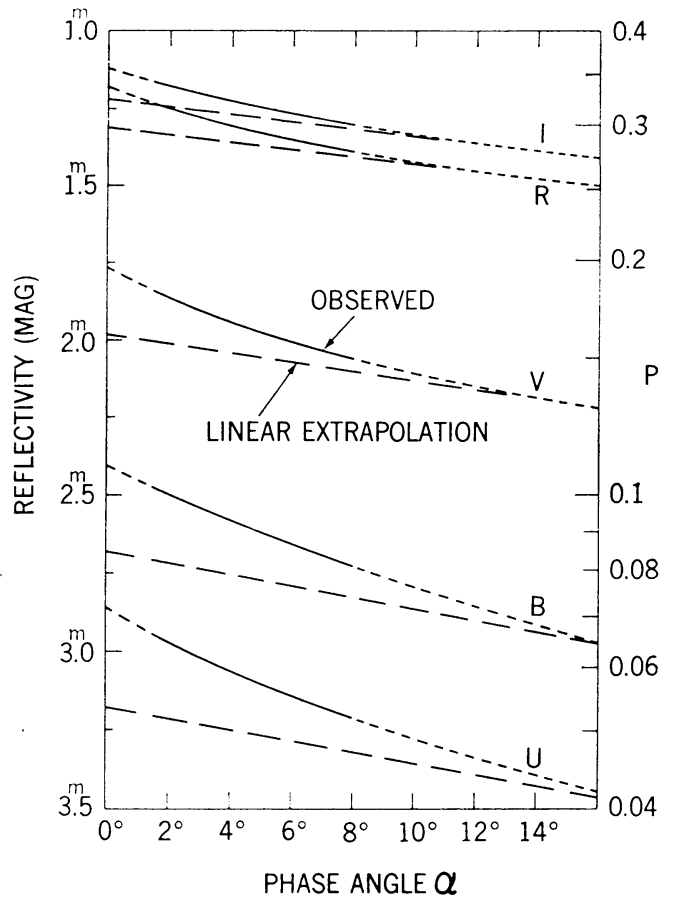

Fig. 1. The Martian opposition effect in five colors, after O'Leary and Rea (1968), adjusted for the color of the sun. The observations (solid lines), which cover $1.2^{\circ} \leqslant \alpha \leqslant 8^{\circ}$, have been fitted to the linear phase functions for $\alpha \geqslant 10^{\circ}$ reported by de Vaucouleurs (1964). Reflectivity is shown on a logarithmic scale on the right and on an equivalent magnitude scale on the left.

The purpose of this study is to investigate the contribution which atmospheric aerosols might make to the Martian opposition effect, under the assumption that the increased enhancement in the shorter wavelengths, where the surface albedo is low, is primarily an atmospheric effect rather than a surface effect.

The Mie scattering theory, which describes single scattering by spherical particles, was used to make light scattering calculations for substances such as ice, water, and solid $\mathrm{CO}_{2}$, which have no significant absorption in the wavelength range under consideration. In addition, calculations were made for highly absorbing materials, such as limonite.

Various submicron particle size distributions were used to compute integrated scattering intensities for these aerosols as a function of phase angle and wavelength. The results indicated that neither substances having a refractive index between 1.20 and 1.50 , which includes ice $(n=1.31)$, water $(1.33)$, and solid $\mathrm{CO}_{2}(1.35$ : Egan and Spagnolo, 1969), nor highly absorbing materials, such as limonite (complex index $\tilde{n}=$ 2.23 $-0.669 i$ at $\lambda=0.365 \mu$ : Egan and Becker, 1969), can produce the opposition effect. Refractive indices of 1.55 to 2.00 , on the other hand, were found to exhibit a definite increase in reflectivity at small phase angles.

A model was constructed to give the total brightness contribution by the surface 
plus atmospheric aerosols. At longer wavelengths, where the Martian albedo is higher and where surface markings are more clearly visible, it is reasonable to assume that the observed brightness comes almost entirely from the surface, and the brightness contribution by aerosols is negligible. As suggested by de Vaucouleurs (1968), we took the lunar photometric function developed by Hapke (1963) and modified it to fit the observed Martian brightness-phase curve at these longer wavelengths. We assumed that the phase curve for the surface would have the same shape in all colors; only the albedo would change. This means that in this model, the surface brightness would increase by $30 \%$ from $16^{\circ}$ to $0^{\circ}$ phase angle at all wavelengths. These assumed surface functions are shown as the thin lower curves for $I, V, B$, and $U$ in Figure 2 ( $R$ has been omitted for simplicity).

The effect of the aerosols was then introduced. The upper heavy solid curves in Figure 2 represent the calculated brightness of surface plus aerosols for refractive index 1.75 and a skewed gaussian-type particle distribution peaked at $0.4 \mu$. At shorter wavelengths, where the albedo and surface contrast are greatly reduced, atmospheric aerosols are seen to play a significant role.

The calculated phase curves are in reasonable agreement with the observations, shown as dashed lines. One should bear in mind, however, that there was a good deal

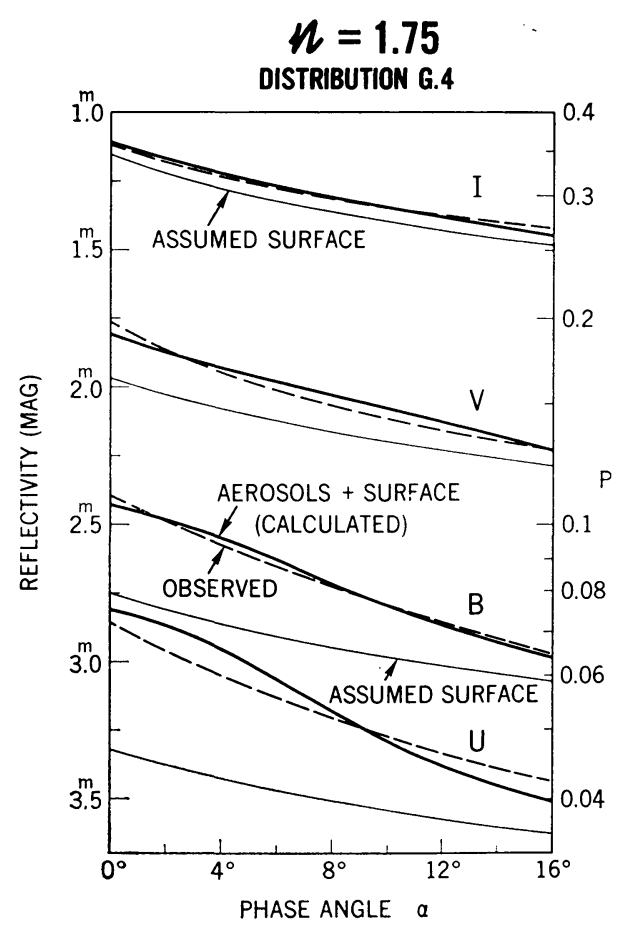

Fig. 2. Comparison of model with Mars observations. For each wavelength, the thin lower curve is the assumed surface reflectivity, the heavy upper curve is the calculated brightness from the surface plus aerosols, and the broken curve is the Martian observational data. 
of arbitrariness in obtaining this fit. It is by no means a unique solution to the problem. It does show, however, that the presence of a small amount of atmospheric aerosols, with the proper index of refraction, could provide the observed increased opposition effect for Mars in the ultraviolet, where the albedo is very low, but at the same time make a negligible contribution in the infrared, where the surface albedo is high.

\section{References}

de Vaucouleurs, G.: 1964, 'Geometric and Photometric Parameters of the Terrestrial Planets', Icarus 3, 187-235.

de Vaucouleurs, G.: 1968, 'On the Opposition Effect of Mars', Icarus 9, 598-599.

Egan, W. G. and Becker, J. F.: 1969, 'Determination of the Complex Index of Refraction of Rocks and Minerals', Appl. Opt. 8, 720-721.

Egan, W. G. and Spagnolo, F. A.: 1969, 'Complex Index of Refraction of Bulk Solid Carbon Dioxide', Appl. Opt. 8, 2359-2360.

Hapke, B. W.: 1963, 'A Theoretical Photometric Function for the Lunar Surface', J. Geophys. Res. 68, 4571-4586.

O'Leary, B. T.: 1967a, 'The Opposition Effect of Mars', Astrophys. J. 149, L147-L149.

O'Leary, B. T.: 1967b, 'Mars: Visible and Near Infrared Studies and the Composition of the Surface', Ph.D. thesis, University of California, Berkeley, Calif., 165 pp.

O'Leary, B. T. and Rea, D. G.: 1968, 'The Opposition Effect of Mars and its Implications', Icarus 9, 405-428. 